\title{
Cell polarity protein Par3 complexes with DNA-PK via Ku70 and regulates DNA double-strand break repair
}

Longhou Fang ${ }^{1,2}$, YiGuo Wang ${ }^{1,2}$, Dan $\mathrm{Du}^{1,2}$, Guang Yang ${ }^{1,2}$, Tim Tak Kwok ${ }^{3}$, Siu Kai Kong ${ }^{3}$, Benjamin Chen ${ }^{4}$, David J Chen ${ }^{4}$, Zhengjun Chen ${ }^{1,5}$

Cell Research (2007) 17:572-574. doi: 10.1038/cr.2007.50; published online 14 June 2007

Correction to: Cell Research (2007) 17:100-116. doi:10.1038/sj.cr.7310145; published online 6 February 2007

The author affiliations were mixed up in the previous published version. The third fund number of National Natural Science Foundation of China in the Acknowledgments was wrong, it should be "30270335". The Shanghai Municipal Council for Science and Technology (No.06DZ22032) was missed in the Acknowledgments. There are some labeling and production errors in Figure 2A, Figure 3B and 3C, Figure 5C, Figure 6B and 6E, Figure 7B and 7D. In Figure 2A, left panel "A431" should be "Par3". In Figure 3B and 3C, "anti-Par3CT" should be "anti-Par3LCT", "GST-Par3CT" should be "GST-Par3LCT". In Figure 5C, the second arrow indicating "Lamin B" should be " $\beta$-tubulin". In Figure 6B right panel, the molecular weight for $\beta$-actin should be " 43 " instead of " 200 ". In Figure 6E, "Par3" should be "Par3i". The molecular weight for the DNA-PKcs panel should be the same as the p-DNA-PKcs. In Figure 7B, the time point "240" in the left panel should be "120"; in the right panel of Figure 7B, the title for the y axis should be "DNA released (\%)". In Figure 7D, the title for the y axis should be "Survival (\%)", and the scale for the y axis should be "100, 10 and 1 ".

These corrections do not affect the conclusions of the study. We apologize for any inconvenience this may have caused.

The corrected author affiliations, Acknowledgments, and Figures are as follows:

${ }^{1}$ Key Laboratory of Proteomics and Laboratory of Molecular Cell Biology, Institute of Biochemistry and Cell Biology, Shanghai Institutes for Biological Sciences, Chinese Academy of Sciences, Shanghai 200031, China ; ${ }^{2}$ Graduate School of the Chinese Academy of Sciences, 320 Yueyang Road, Shanghai 200031, China; ${ }^{3}$ Department of Biochemistry, The Chinese University of Hong Kong, Hong Kong SAR, China; ${ }^{4}$ Department of Radiation Oncology, Division of Molecular Radiation Biology, University of Texas Southwestern Medical Center, Dallas, TX, USA; ${ }^{5}$ SHARF Laboratory, Institute of Biochemistry and Cell Biology, Shanghai Institutes for Biological Sciences, Chinese Academy of Sciences, Shanghai 200031, China

\section{Acknowledgments}

We thank Dr Yingjie Wu for critical reading of the manuscript. We also thank Drs Stephen Jackson, Peter Ahnesorg, Terry Lechler, Gerard Drewes for helpful communication. We thank Dr Ian G Macara for providing the Par3 plasmid. We thank Dr Hu Zhou for the MS experiment. This work was supported by the grants from National Natural Science Foundation of China (No.30170208, 30623003 and 30270335); from the Ministry of Science and Technology, China (No.2001AA233031 and No.2001CB510205); from the Shanghai Municipal Council for Science and Technology (No.06DZ22032), and from US NIH (CA50519) (to DJ Chen). 
Figure 2A

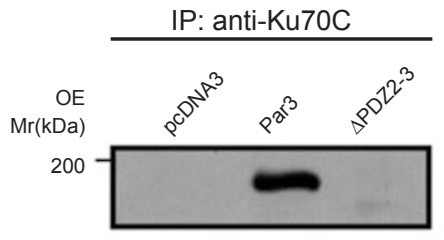

IB: anti-Par3

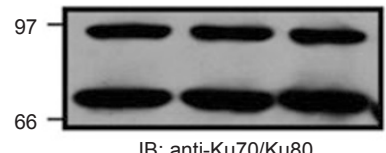

IB: anti-Ku70/Ku80
Figure 5C

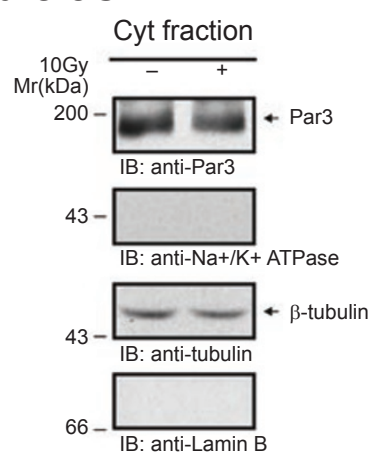

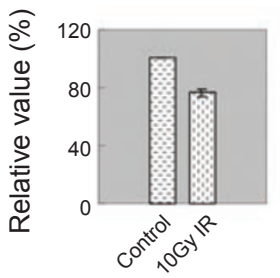

Figure 3

B Caco-2 cells

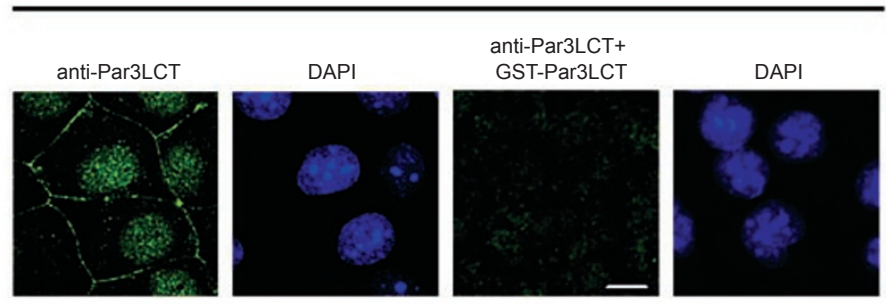

C

HeLa cells

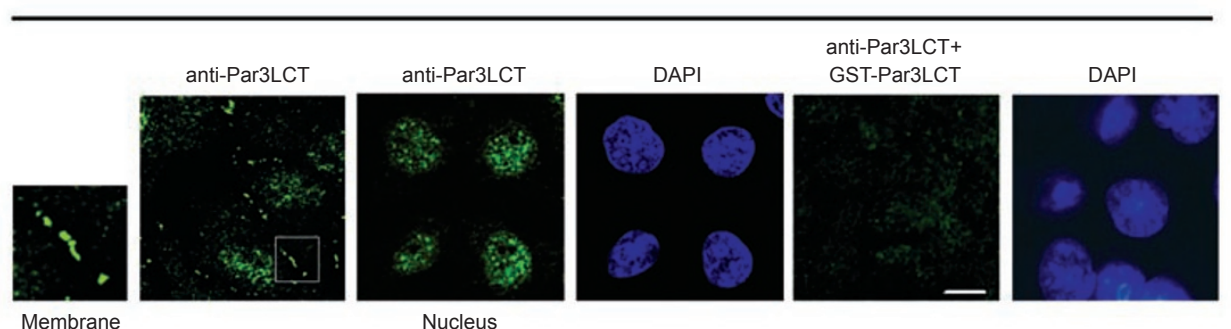

Figure 6B
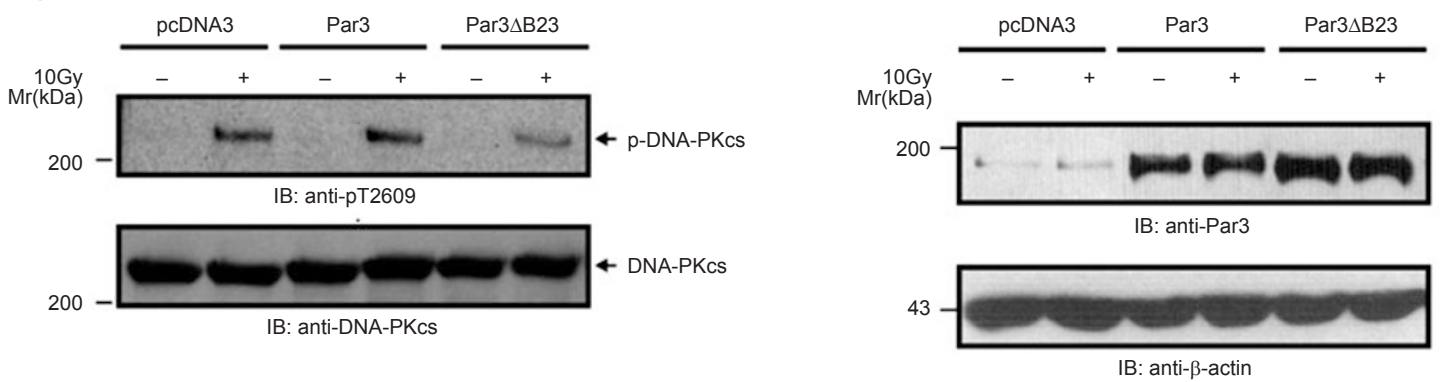
Figure 6E
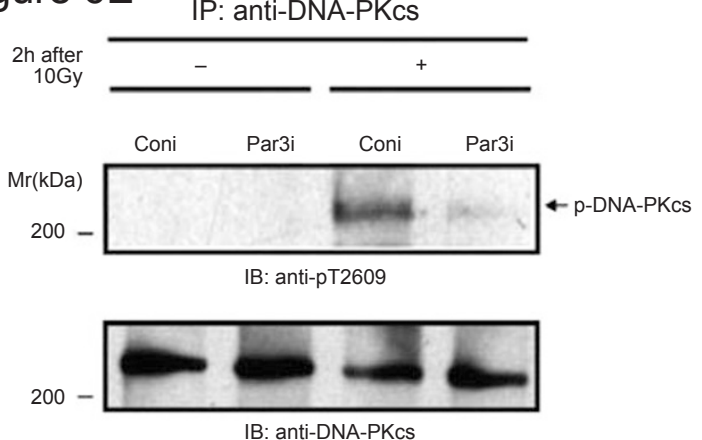

IB: anti-DNA-PKcs

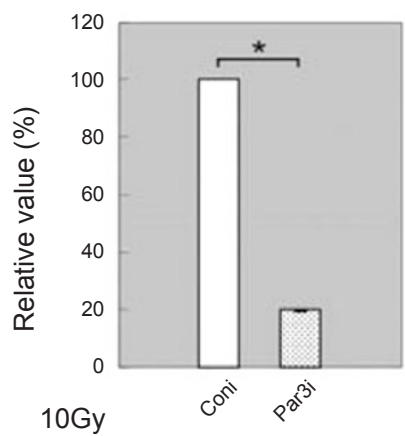

\section{Figure 7}

B

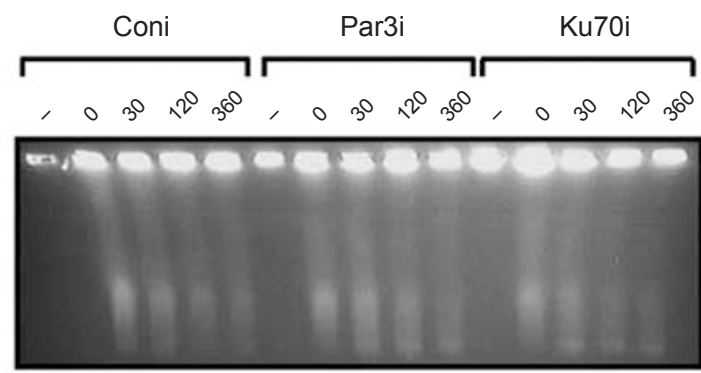

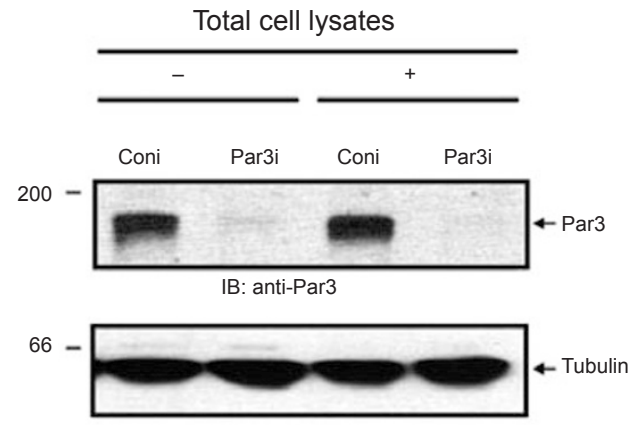

IB: anti-tubulin

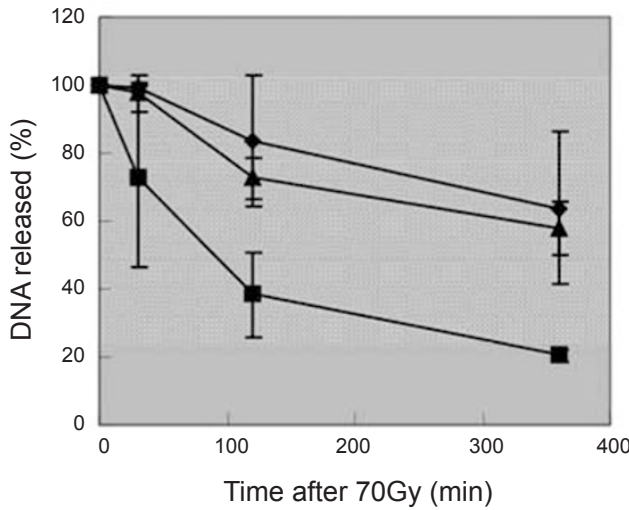

D

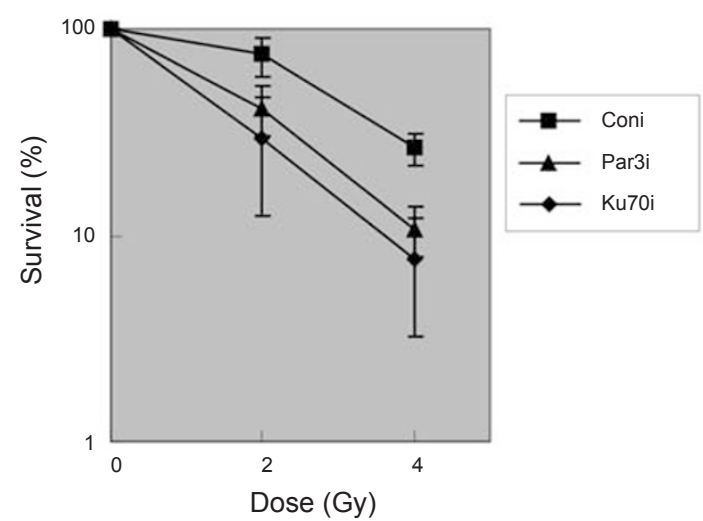

